ESAIM: M2AN 47 (2013) 109-123

DOI: $10.1051 / \mathrm{m} 2 \mathrm{an} / 2012021$
ESAIM: Mathematical Modelling and Numerical Analysis

www.esaim-m2an.org

\title{
AN ANALYSIS OF THE BOUNDARY LAYER IN THE 1D SURFACE CAUCHY-BORN MODEL *
}

\author{
Kavinda Jayawardana ${ }^{1}$, Christelle MordacQ ${ }^{2}$, \\ Christoph Ortner ${ }^{3}$ and Harold S. PARK ${ }^{4}$
}

\begin{abstract}
The surface Cauchy-Born (SCB) method is a computational multi-scale method for the simulation of surface-dominated crystalline materials. We present an error analysis of the SCB method, focused on the role of surface relaxation. In a linearized 1D model we show that the error committed by the SCB method is $\mathcal{O}(1)$ in the mesh size; however, we are able to identify an alternative "approximation parameter" - the stiffness of the interaction potential - with respect to which the relative error in the mean strain is exponentially small. Our analysis naturally suggests an improvement of the SCB model by enforcing atomistic mesh spacing in the normal direction at the free boundary. In this case we even obtain pointwise error estimates for the strain.
\end{abstract}

Mathematics Subject Classification. 70C20, 70-08, 65N12, 65N30.

Received December 5, 2011. Revised April 6, 2012.

Published online July 31, 2012.

\section{INTRODUCTION}

Miniaturization of materials to the nanometer scale has led to unexpected and often enhanced mechanical properties that are not found in corresponding bulk materials $[6,33]$. This size-dependence has been experimentally observed to begin around a scale of about $100 \mathrm{~nm}$ [27]. A fully atomistic simulation of a nanostructure of this size would require on the order of $10^{8}$ atoms, which motivates the need for computationally efficient multiscale methods.

The underlying cause for the size-dependent mechanical properties is that surface atoms have fewer bonding neighbours, or a coordination number reduction, as compared to atoms that lie within the material bulk. This results in the elastic properties of surfaces being different from those of an idealized bulk material [27], which

Keywords and phrases. Surface-dominated materials, surface Cauchy-Born rule, coarse-graining.

* KJ and CM were supported by undergraduate vacation bursaries at the Oxford Centre for Nonlinear PDE. CO was supported by the EPSRC Grant EP/H003096 "Analysis of Atomistic-to-Continuum Coupling Methods". HP was supported by NSF grants CMMI-0750395 and CMMI-1036460.

1 Department of Mathematics, University College London, Gower Street, WC1E 6BT London, UK. k.guruge@ucl.ac.uk

2 École Normale Supérieure de Cachan, Antenne de Bretagne, Avenue Robert Schuman, 35170 Bruz, France. Christelle.Mordacq@gmail.com

3 Mathematics Institute, Zeeman Building, University of Warwick, CV4 7AL Coventry, UK. c.ortner@warwick.ac.uk

4 Boston University, Department of Mechanical Engineering, 730 Commonwealth Avenue, ENA 212, Boston, 02215 MA, USA.

parkhs@acs.bu.edu 
becomes important with decreasing structural size and increasing surface area to volume ratio [6]. Additionally, nanoscale surface stresses [5], which also arise from the coordination number reduction of surface atoms [35], cause deformation of not only the surfaces, but also the underlying bulk [17], and can result in unique physical properties such as phase transformations [7], or shape memory and pseudoelasticity effects in FCC nanowires that are not observed in the corresponding bulk material $[18,25]$.

To study surface-dominated nanostructures, Park et al. recently developed the surface Cauchy-Born (SCB) model $[22,23,26]$. The idea is to seek an energy functional of the form

$$
E^{\mathrm{scb}}(y)=\int_{\Omega} W(\nabla y) \mathrm{d} x+\int_{\partial \Omega} \gamma(\nabla y, \nu) \mathrm{d} s
$$

where $\Omega \subset \mathbb{R}^{3}$ is an elastic body, $y: \Omega \rightarrow \mathbb{R}^{3}$ a deformation field, $W$ the bulk stored energy function, and $\gamma$ a surface stored energy function. The potentials $W, \gamma$ are chosen such that $W(\mathrm{~F})$ denotes the energy per unit volume in an infinite crystal under the deformation $y(x)=\mathrm{F} x$, while $\gamma(\mathrm{F}, \nu)$ is the surface energy per unit area of a half-space with surface normal $\nu$, under the deformation $y(x)=\mathrm{F} x$. Thus, $W$ and $\gamma$ are derived from the underlying atomistic model. For $W$ this is a well-understood idea $[1,10,12,19]$; the novel approach in the SCB method is to apply the same principle to the surface energy potential. We note, however, that a surface contribution as in (1.1) was previously derived in [1], as the first-order expansion of an atomistic model with pair interactions. A more explicit form of the surface energy contribution in polygonal domains is given in [30].

In contrast to the SCB method, most computational models (see, e.g., $[13,15,40]$ ) are based upon a finite element discretization of the governing surface elasticity equations of Gurtin and Murdoch [14], where the constitutive relation for the surface is linearly elastic or uses standard hyperelastic strain energy functions [16].

The SCB model was successfully applied to various nanomechanical boundary value problems, including thermomechanical coupling [38], resonant frequencies, and elucidating the importance of nonlinear, finite deformation kinematics on the resonant frequencies of both FCC metal [24] and silicon nanowires [20, 21], bending of FCC metal nanowires [39], and electromechanical coupling in surface-dominated nanostructures [28]. A further application that we aim to pursue in future work is the simulation of cracks in bulk crystalline materials (see [2-4] for related works), which requires the accurate description of the crack surface; in more than one dimension this will require the development of a coupling mechanism at crystal surfaces.

The purpose of the present work is to initiate a mathematical analysis of the accuracy of the SCB method. We focus on the simplest setting where the only effect is a surface relaxation in normal direction. While the SCB model does include surface physics that are neglected in the standard Cauchy-Born (CB) model, due to employing a coarse finite element discretisation it does not resolve the resulting boundary layer; see the numerical results in [11] as well as Figure 1 for a 1D toy model demonstrating this. It is therefore a priori unclear to what extent the SCB improves upon the CB model. Figure 1 suggests that, while the error in the displacement and displacement gradient is indeed of order $\mathcal{O}(1)$ in the boundary layer, the displacement error at finite element nodes is visually negligable, which would imply that the SCB model approximates the mean strain (and possibly other averaged quantities) to a much higher degree of accuracy. This was indeed observed in extensive numerical tests presented in $[11,22,23]$.

There is no traditional discretisation or approximation parameter in this model with respect to which we might try to explain this effect. Instead, our analysis measures the SCB error in terms of the stiffness of the interaction potential. This enables us to identify a suitable asymptotic limit for our analysis on a linearized model problem. We confirm the analytical predictions with numerical experiments on the fully nonlinear problem in $1 \mathrm{D}$ and a periodic semi-infinite $2 \mathrm{D}$ domain.

To the best of our knowledge, our work presents the first approximation error results for the SCB method. Although our analysis is elementary, it makes three novel contributions: (1) we show that the "correct" approximation parameter is the stiffness of the interaction potential (however, Theil [37] uses similar ideas for an analysis of surface relaxation); and (2) we show that the mean strain (which is an important quantity of interest) has a much lower relative error than the strain field. (3) Our results show how to substantially improve the accuracy 

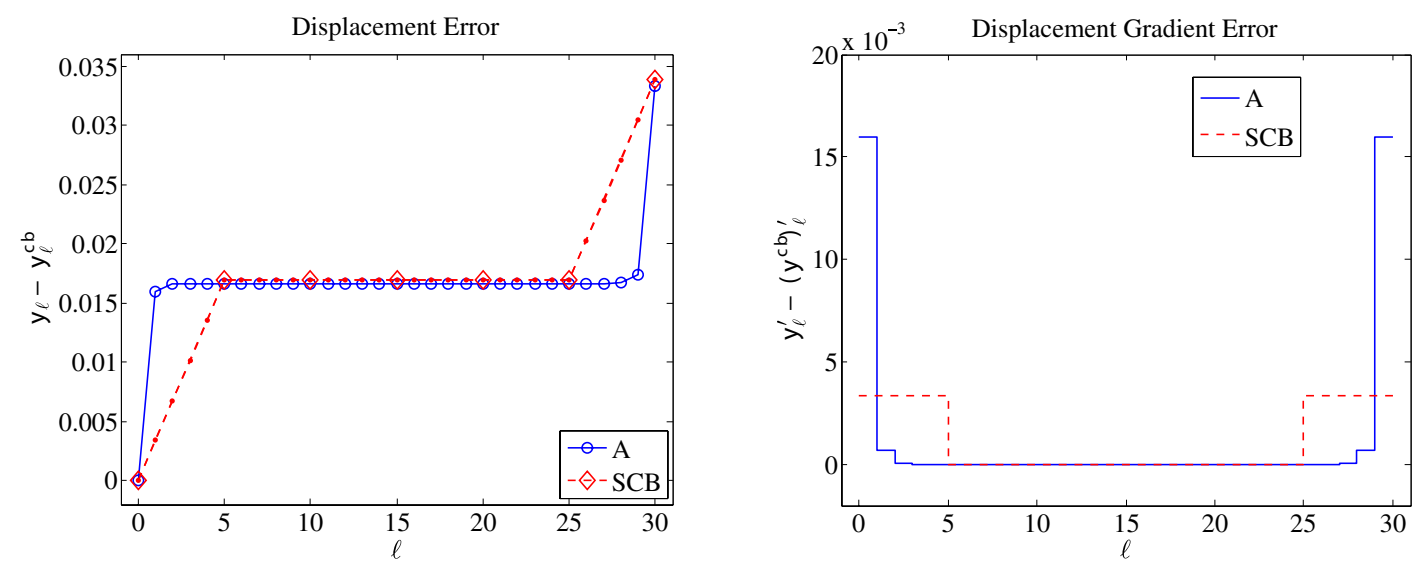

FiguRE 1. Displacements and displacement gradients of an atomistic solution and a surface Cauchy-Born solution, relative to the bulk Cauchy-Born solution, for the 1D model problem described in Sections 2.1 and 2.2. We observe unexpectedly high accuracy of the displacement at the finite element nodes (marked by diamonds) despite a large error in the displacement gradient.

of the SCB method at moderate additional computational cost. Finally, we hope that this work will stimulate further research on computationally efficient multiscale methods for surface-dominated nanostructures.

The issues we address here are closely related to the classical problem of numerical methods for resolving boundary layers [29]. The main difference in our case is the discrete setting which does not give us the opportunity to let the mesh-size tend to zero. For a mathematical analysis of thin atomistic structures, surface energies and surface relaxation we refer to $[3,31,32,37]$ and references therein. Our work also draws inspiration from $[8,9]$ where a similar linearised model problem is used to analyze the accuracy of atomistic-to-continuum coupling methods.

Finally, we remark that we restrict our analysis to a specific choice of the interaction potential, to make it as simple as possible. The choice of the Morse potential is motivated by the explicit occurance of a model parameter that controls the "stiffness" of the potential. Qualitatively, we expect that our conclusions apply to any pair interaction model where the potential has a high second derivative in its minimum and decays rapidly with increasing bond length.

\section{ANALYSIS OF A 1D MODEL PROBLEM}

\subsection{Atomistic model}

We consider a semi-infinite chain of atoms with reference positions $\ell \in \mathbb{N}$, and deformed positions $y_{\ell}, \ell \in$ $\mathbb{N}$. We assume that the chain interacts through second-neighbour Morse pair interaction. Hence, a deformed configuration $y$ has energy (defined formally for the moment; $c f$. Prop. 2.1)

$$
E^{\mathrm{a}}(y):=\sum_{\ell=0}^{\infty}\left[\phi\left(y_{\ell+1}-y_{\ell}\right)+\phi\left(y_{\ell+2}-y_{\ell}\right)\right]
$$

where $\phi$ is a shifted Morse potential with stiffness parameter $\alpha>0$ and potential minimum $r_{0}>0$,

$$
\phi(r)=\exp \left(-2 \alpha\left(r-r_{0}\right)\right)-2 \exp \left(-\alpha\left(r-r_{0}\right)\right)-\phi_{0},
$$

where $\phi_{0}$ is chosen to that $W(1)=0$, where

$$
W(r):=\phi(r)+\phi(2 r),
$$


$r_{0}$ is defined such that $W^{\prime}(1)=0$,

$$
r_{0}=1+\frac{1}{\alpha} \log \left(\frac{1+2 e^{-\alpha}}{1+2 e^{-2 \alpha}}\right)
$$

and $\alpha \geq 1+\sqrt{3}$ remains a free parameter. This restriction on $\alpha$ ensures that $\phi^{\prime \prime}(2) \leq 0$, which will be convenient in the analysis. The shift of the potential by $\phi_{0}$ ensures that $E^{\mathrm{a}}(\mathrm{id})$ is finite.

The potential $W$ is called the Cauchy-Born stored energy density. We have chosen the parameters in the Morse potential so that 1 is the minimizer of $W$.

Since $E^{\mathrm{a}}$ is translation invariant, it is convenient to fix $y_{0}=0$. In that case, $y_{\ell}$ is completely determined by the forward differences $y_{\ell}^{\prime}:=y_{\ell+1}-y_{\ell}$. Hence we change coordinates from the deformation $y_{\ell}$ to the displacement gradient $u_{\ell}:=y_{\ell}^{\prime}-1$, and rewrite $E^{\mathrm{a}}$ as

$$
E^{\mathrm{a}}(u):=\sum_{\ell=0}^{\infty}\left[\phi\left(1+u_{\ell}\right)+\phi\left(2+u_{\ell}+u_{\ell+1}\right)\right] .
$$

The next result establishes that $E^{\mathrm{a}}$ is well-defined in a suitable function space setting; the proof is given in the appendix.

Proposition 2.1. $E^{\mathrm{a}}$ is well-defined and twice Fréchet differentiable in $\ell^{1}(\mathbb{N})$ with first and second variations given by

$$
\begin{aligned}
\left\langle\delta E^{\mathrm{a}}(u), v\right\rangle & =\sum_{\ell=0}^{\infty}\left[\phi^{\prime}\left(1+u_{\ell}\right) v_{\ell}+\phi^{\prime}\left(2+u_{\ell}+u_{\ell+1}\right)\left(v_{\ell}+v_{\ell+1}\right)\right] \\
\left\langle\delta^{2} E^{\mathrm{a}}(u) v, w\right\rangle & =\sum_{\ell=0}^{\infty}\left[\phi^{\prime \prime}\left(1+u_{\ell}\right) v_{\ell} w_{\ell}+\phi^{\prime \prime}\left(2+u_{\ell}+u_{\ell+1}\right)\left(v_{\ell}+v_{\ell+1}\right)\left(w_{\ell}+w_{\ell+1}\right)\right] .
\end{aligned}
$$

\subsection{The Cauchy-Born and surface Cauchy-Born models}

The Cauchy-Born approximation is designed to model elastic bulk behaviour in crystals. The stored energy density is chosen so that the Cauchy-Born energy is exact under homogeneous deformations in the absence of defects (such as surfaces). For the 1D model (2.1) this yields (formally for the moment; cf. Prop. 2.2)

$$
E^{\mathrm{cb}}(y):=\int_{0}^{\infty} W\left(y^{\prime}\right) \mathrm{d} x
$$

or equivalently, written in terms of the displacement gradient $u=y^{\prime}-1$,

$$
E^{\mathrm{cb}}(u)=\int_{0}^{\infty} W(1+u) \mathrm{d} x
$$

where $W(r)=\phi(r)+\phi(2 r)$ was already defined in $(2.3)$.

We consider a $\mathrm{P}_{1}$ finite element discretisation of the Cauchy-Born model. Let $X_{h}:=\left\{X_{0}, X_{1}, \ldots\right\} \subset \mathbb{N}$ be a strictly increasing sequence of grid points with $X_{0}=0$, and let $h_{j}:=X_{j+1}-X_{j} \in \mathbb{N}$. A $\mathrm{P}_{1}$ discretisation of $y$ corresponds to a $\mathrm{P}_{0}$ discretisation of the displacement gradient $u$, hence we define for $\left(U_{j}\right)_{j=0}^{\infty} \subset \mathbb{R}$, where $U_{j}$ denotes the displacement gradient in the element $\left(X_{j}, X_{j+1}\right)$,

$$
E_{h}^{\mathrm{cb}}(U):=\sum_{j=0}^{\infty} h_{j} W\left(1+U_{j}\right) .
$$




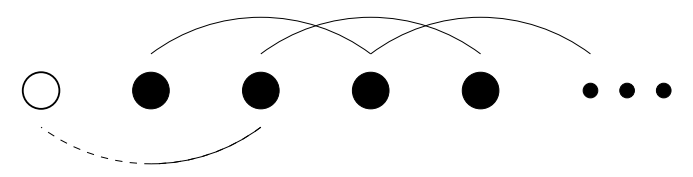

Figure 2. Visualisation of (2.7): the bond at the left-hand end of the graph is counted half in the Cauchy-Born model, even though it does not exist in the atomistic model, hence it gives a contribution $-\frac{1}{2} \phi\left(2 y^{\prime}(0)\right)$ to the surface energy.

The Cauchy-Born approximation commits an error at the crystal surface, which the surface Cauchy-Born (SCB) approximation aims to rectify. The idea of the SCB method (in our 1D setting) is to define (again only formally; cf. Prop. 2.2)

$$
E^{\mathrm{scb}}(y):=\int_{0}^{\infty} W\left(y^{\prime}\right) \mathrm{d} x+\gamma\left(y^{\prime}(0)\right),
$$

and choose $\gamma$ such that the energy is exact under homogeneous deformations, which yields the formula

$$
\gamma(F):=-\frac{1}{2} \phi(2 F)
$$

see also Figure 2. Converting to the displacement gradient coordinate discretised by the $\mathrm{P}_{0}$ finite element method we obtain

$$
E_{h}^{\mathrm{scb}}(U):=E_{h}^{\mathrm{cb}}(U)+\gamma\left(1+U_{0}\right)
$$

The next result establishes that $E_{h}^{\mathrm{cb}}$ and $E_{h}^{\mathrm{scb}}$ are well-defined in a suitable function space setting; the proof is given in the appendix.

Proposition 2.2. $E_{h}^{\mathrm{cb}}$ and hence $E_{h}^{\mathrm{scb}}$ are well-defined and twice Fréchet differentiable in the weighted space $\ell_{h}^{1}\left(X_{h}\right):=\left\{V=\left(V_{j}\right)_{j=0}^{\infty}\right\}$ equipped with the norm

$$
\|V\|_{\ell_{h}^{1}}:=\sum_{j=0}^{\infty} h_{j}\left|V_{j}\right| .
$$

The first and second variations of $E_{h}^{\mathrm{scb}}$ are given, respectively, by

$$
\begin{aligned}
\left\langle\delta E_{h}^{\mathrm{scb}}(U), V\right\rangle & =\sum_{j=0}^{\infty} h_{j} W^{\prime}\left(1+U_{j}\right) V_{j}+\gamma^{\prime}\left(1+U_{0}\right) V_{0}, \quad \text { and } \\
\left\langle\delta^{2} E_{h}^{\mathrm{scb}}(U) V, W\right\rangle & =\sum_{j=0}^{\infty} h_{j} W^{\prime \prime}\left(1+U_{j}\right) V_{j} W_{j}+\gamma^{\prime \prime}\left(1+U_{0}\right) V_{0} W_{0} .
\end{aligned}
$$

\subsection{Analysis of the linearized models}

The parameter $r_{0}$ for the Morse potential was chosen so that 1 is the minimizer of the Cauchy-Born stored energy function, which implies that

$$
U_{j}^{\mathrm{cb}}:=0, \text { for } j=0,1, \ldots
$$

is the ground state of $E_{h}^{\mathrm{cb}}$. More generally, $u^{\mathrm{cb}}:=(0)_{\ell=0}^{\infty}$ gives the Cauchy-Born ground state. We now consider linearisations of $E_{h}^{\mathrm{scb}}$ and $E^{\mathrm{a}}$ about the Cauchy-Born state: $\delta E(0)+\delta^{2} E(0) u=0$, where $E \in\left\{E^{\mathrm{a}}, E_{h}^{\mathrm{scb}}\right\}$.

From Proposition 2.2 we obtain the linearised optimality condition for $E_{h}^{\text {scb }}$,

$$
\gamma^{\prime}(1)+\left(h_{0} W^{\prime \prime}(1)+\gamma^{\prime \prime}(1)\right) U_{0}=0, \quad \text { and } \quad h_{j} W^{\prime \prime}(1) U_{j}=0 \text { for } j=1,2, \ldots,
$$


which gives the linearised surface Cauchy-Born solution

$$
U_{0}^{\mathrm{scb}}=\frac{-\gamma^{\prime}(1)}{h_{0} W^{\prime \prime}(1)+\gamma^{\prime \prime}(1)}, \quad \text { and } \quad U_{j}^{\text {scb }}=0, \quad \text { for } j=1,2, \ldots
$$

From Proposition 2.1 we obtain the linearised optimality condition for the atomistic model $E^{\mathrm{a}}$,

$$
\begin{aligned}
\phi^{\prime}(1)+\phi^{\prime \prime}(1) u_{0}+\phi^{\prime}(2)+\phi^{\prime \prime}(2)\left(u_{0}+u_{1}\right) & =0, \\
\phi^{\prime}(1)+\phi^{\prime \prime}(1) u_{j}+2 \phi^{\prime}(2)+\phi^{\prime \prime}(2)\left(u_{j-1}+2 u_{j}+u_{j+1}\right) & =0, \quad j \geq 1,
\end{aligned}
$$

which, using the fact that $\phi^{\prime}(1)+2 \phi^{\prime}(2)=W^{\prime}(1)=0$ can be rewritten in the form

$$
\begin{aligned}
{\left[\phi^{\prime \prime}(1)+\phi^{\prime \prime}(2)\right] u_{0}+\phi^{\prime \prime}(2) u_{1} } & =\phi^{\prime}(2), \\
\phi^{\prime \prime}(2) u_{\ell-1}+\left[\phi^{\prime \prime}(1)+2 \phi^{\prime \prime}(2)\right] u_{\ell}+\phi^{\prime \prime}(2) u_{\ell+1} & =0, \quad \ell \geq 1 .
\end{aligned}
$$

This finite difference equation can be easily solved explicitly, which yields the solution

$$
u_{\ell}^{\mathrm{a}}:=\frac{\phi^{\prime}(2) \lambda^{\ell}}{\phi^{\prime \prime}(1)+\phi^{\prime \prime}(2)(1+\lambda)}, \quad \text { where } \quad \lambda=\frac{\sqrt{1+4 \frac{\phi^{\prime \prime}(2)}{\phi^{\prime \prime}(1)}}-1-2 \frac{\phi^{\prime \prime}(2)}{\phi^{\prime \prime}(1)}}{2 \frac{\phi^{\prime \prime}(2)}{\phi^{\prime \prime}(1)}}
$$

is the unique solution in $(0,1)$ of the characteristic equation

$$
\phi^{\prime \prime}(2) \lambda^{2}+\left[\phi^{\prime \prime}(1)+2 \phi^{\prime \prime}(2)\right] \lambda+\phi^{\prime \prime}(2)=0 .
$$

(Note that there is also an exponentially growing solution, but it does not have finite energy).

Since the expressions for (2.9) and (2.10) are somewhat bulky we expand them in the stiffness parameter $\alpha$ of the Morse potential (2.2). The elementary proof is postponed to the appendix.

Our rationale for expanding in this parameter is as follows:

1. Interactions in crystalline solids are typically fairly stiff; e.g., the Lennard-Jones case corresponds roughly to $\alpha$ between 4.5 and 5 . This may seem only moderate, but note that our estimates are in terms of $e^{-\alpha}$, and that $e^{-5} \approx 0.007$.

2. All three models (atomistic, $\mathrm{CB}, \mathrm{SCB}$ ) formally coincide in the limit $\alpha \rightarrow \infty$ since in the elastic regime the second neighbour interaction tends to zero while the nearest neighbour potential tends to a sticky potential.

3. It is common for asymptotic expansions of this type to be accurate in a much wider range than expected; hence we hope that our results will also yield useful predictions for moderate $\alpha$.

Proposition 2.3. Asymptotically as $\alpha \rightarrow \infty$ we have the expansions

$$
\begin{aligned}
U_{0}^{\mathrm{scb}} & =\frac{e^{-\alpha}}{h_{0} \alpha}\left[1-\left(1+\frac{2}{h_{0}}\right) e^{-\alpha}+\mathcal{O}\left(e^{-2 \alpha}\right)\right], \quad \text { and } \\
u_{0}^{\mathrm{a}} & =\frac{e^{-\alpha}}{\alpha}\left[1-4 e^{-\alpha}+\mathcal{O}\left(e^{-2 \alpha}\right)\right] .
\end{aligned}
$$

Remark 2.4. The asymptotic expansions (2.11) and (2.12) justify a posteriori the linearisation since they show that the displacements from the Cauchy-Born state are indeed small in the limit as $\alpha \rightarrow \infty$.

\subsection{Error estimates}

We first note that each $\mathrm{P}_{0}$ function $U=\left(U_{j}\right)_{j=0}^{\infty}$ can be understood as a lattice function $u=\left(u_{\ell}\right)_{\ell=0}^{\infty}$ through the interpolation

$$
u_{\ell}=U_{j} \quad \text { for } \ell=X_{j}, \ldots, X_{j+1}-1, \quad j \in \mathbb{N} .
$$


With this interpolation we obtain $u^{\mathrm{cb}}=0$ and $u^{\mathrm{scb}}$ from the linearized CB and SCB solutions $U^{\mathrm{cb}}$ and $U^{\mathrm{scb}}$, given in (2.9).

We are interested in the improvement the SCB model gives over the pure Cauchy-Born model, that is, we wish to measure the relative errors

$$
\operatorname{Err}_{p}:=\frac{\left\|u^{\mathrm{scb}}-u^{\mathrm{a}}\right\|_{\ell^{p}}}{\left\|u^{\mathrm{cb}}-u^{\mathrm{a}}\right\|_{\ell^{p}}}=\frac{\left\|u^{\mathrm{scb}}-u^{\mathrm{a}}\right\|_{\ell^{p}}}{\left\|u^{\mathrm{a}}\right\|_{\ell^{p}}} .
$$

Of particular interest are the uniform error $\operatorname{Err}_{\infty}$ and the error in the energy-norm $\operatorname{Err}_{2}$. We shall consider two separate cases: $h_{0}>1$ and $h_{0}=1$.

Proposition 2.5 (strain error). Let $p \in[1, \infty]$ and $h_{0}>1$, then

$$
\operatorname{Err}_{p}=C_{p}+\mathcal{O}\left(e^{-\alpha}\right)
$$

where $\frac{1}{2} \leq C_{p} \leq 2$. If $h_{0}=1$, then

$$
\operatorname{Err}_{p}=2^{1 / p} e^{-\alpha}+\mathcal{O}\left(e^{-2 \alpha}\right) .
$$

Proof. We consider the case $h_{0}=1$ first. In that case (2.10) gives us

$$
\left(\sum_{\ell=1}^{\infty}\left|u_{\ell}^{\mathrm{scb}}-u_{\ell}^{\mathrm{a}}\right|^{p}\right)^{1 / p}=\left(\sum_{\ell=1}^{\infty}\left|u_{\ell}^{\mathrm{a}}\right|^{p}\right)^{1 / p}=\lambda u_{0}^{\mathrm{a}}\left(1-\lambda^{p}\right)^{-1 / p}
$$

and similarly, $\left\|u^{\mathrm{a}}\right\|_{\ell^{p}}=u_{0}^{\mathrm{a}}\left(1-\lambda^{p}\right)^{-1 / p}$. Using the asymptotic expansions (A.5) for $\lambda$ it is straightforward to show that

$$
\left(1-\lambda^{p}\right)^{-1 / p}=1+\mathcal{O}(\lambda)=1+\mathcal{O}\left(e^{-\alpha}\right)
$$

hence employing also (2.12) we obtain

$$
\left\|u^{\mathrm{a}}\right\|_{\ell^{p}}=\frac{e^{-\alpha}}{\alpha}+\mathcal{O}\left(\frac{e^{-2 \alpha}}{\alpha}\right), \quad \text { and }\left(\sum_{\ell=1}^{\infty}\left|u_{\ell}^{\mathrm{scb}}-u_{\ell}^{\mathrm{a}}\right|^{p}\right)^{1 / p}=\frac{e^{-2 \alpha}}{\alpha}+\mathcal{O}\left(\frac{e^{-3 \alpha}}{\alpha}\right) .
$$

For $\ell=0$, since $h_{0}=1$, we have

$$
\begin{aligned}
\left|u_{0}^{\mathrm{scb}}-u_{0}^{\mathrm{a}}\right| & =\mid \frac{e^{-\alpha}}{\alpha}\left[1-3 e^{-\alpha}+\mathcal{O}\left(e^{-2 \alpha}\right)\right]-\frac{e^{-\alpha}}{\alpha}\left[1-4 e^{-\alpha}+\mathcal{O}\left(e^{-2 \alpha}\right)\right] \\
& =\frac{e^{-2 \alpha}}{\alpha}+\mathcal{O}\left(\frac{e^{-3 \alpha}}{\alpha}\right) .
\end{aligned}
$$

Combined with (2.15) this gives

$$
\operatorname{Err}_{p}=\frac{\left\|u^{\mathrm{a}}-u^{\mathrm{scb}}\right\|_{\ell^{p}}}{\left\|u^{\mathrm{a}}\right\|_{\ell^{p}}}=\frac{2^{1 / p} \frac{e^{-2 \alpha}}{\alpha}+\mathcal{O}\left(\frac{e^{-3 \alpha}}{\alpha}\right)}{\frac{e^{-\alpha}}{\alpha}+\mathcal{O}\left(\frac{e^{-2 \alpha}}{\alpha}\right)}=2^{1 / p} e^{-\alpha}+\mathcal{O}\left(e^{-2 \alpha}\right),
$$

which concludes the proof of (2.14).

In the case $h_{0}>1$ the convenient cancellation of first-order terms in $u_{0}^{\text {scb }}-u_{0}^{\text {a }}$ does not occur. Instead, using (2.15) we obtain

$$
\left\|u^{\mathrm{a}}-u^{\mathrm{scb}}\right\|_{\ell^{p}}=\frac{e^{-\alpha}}{\alpha}\left(\left|1-\frac{1}{h_{0}}\right|^{p}+\sum_{\ell=1}^{X_{1}-1}\left|\frac{1}{h_{0}}\right|^{p}\right)^{1 / p}+\mathcal{O}\left(\frac{e^{-2 \alpha}}{\alpha}\right) .
$$

This immediately gives (2.13). 
We see from (2.13) that if we use a coarse finite element mesh up to the boundary, then the error in the displacement gradient will be typically of the order $50 \%$ or more. By contrast, if we refine the finite element mesh to atomistic precision at the boundary then the relative error is exponentially small in the stiffness parameter $\alpha$.

The quantity $\operatorname{Err}_{p}$ measures the error in a pointwise sense. However, in some cases we are only interested in correctly reproducing certain macroscopic quantities such as the mean strain error

$$
\overline{\operatorname{Err}}:=\left|\frac{\sum_{\ell=0}^{\infty}\left(u_{\ell}^{\mathrm{scb}}-u_{\ell}^{\mathrm{a}}\right)}{\sum_{\ell=0}^{\infty} u_{\ell}^{\mathrm{a}}}\right| .
$$

Note that, up to higher order terms, this error also bounds the error in the displacements at the finite element nodes, which we observed in Figure 1 to be much smaller than the strain error. We also remark that, even though the mean strain is a macroscopic quantity, the fact that domain is infinite shows that the macro-scale (i.e. the domain size) does not play a role. As a matter of fact, all displacements are highly localised near the domain boundary.

In the following result we confirm that, indeed, the mean strain error is an order of magnitude smaller than the pointwise strain error.

Proposition 2.6 (mean strain error). Asymptotically as $\alpha \rightarrow \infty$, the mean strain error satisfies

$$
\overline{\operatorname{Err}}=2\left(1-\frac{1}{h_{0}}\right) e^{-\alpha}+\mathcal{O}\left(e^{-2 \alpha}\right) .
$$

Proof. We first compute the mean strains in the atomistic and the SCB models. For the atomistic model we have

$$
\bar{u}^{\mathrm{a}}:=\sum_{\ell=0}^{\infty} u_{\ell}^{\mathrm{a}}=\frac{u_{0}^{\mathrm{a}}}{1-\lambda} .
$$

Since $(1-\lambda)^{-1}=1+e^{-\alpha}+\mathcal{O}\left(e^{-2 \alpha}\right)$ we obtain

$$
\bar{u}^{\mathrm{a}}=\frac{e^{-\alpha}}{\alpha}\left[\left(1-4 e^{-\alpha}\right)\left(1+e^{-\alpha}\right)+\mathcal{O}\left(e^{-2 \alpha}\right)\right]=\frac{e^{-\alpha}}{\alpha}\left[1-3 e^{-\alpha}+\mathcal{O}\left(e^{-2 \alpha}\right)\right] .
$$

For the SCB model, we have

$$
\bar{u}^{\mathrm{scb}}=\sum_{j=0}^{\infty} h_{j} U_{j}^{\mathrm{scb}}=h_{0} U_{0}^{\mathrm{scb}}=\frac{e^{-\alpha}}{\alpha}\left[1-\left(1+\frac{2}{h_{0}}\right) e^{-\alpha}+\mathcal{O}\left(e^{-2 \alpha}\right)\right],
$$

and hence the error is given by

$$
\bar{u}^{\mathrm{scb}}-\bar{u}^{\mathrm{a}}=2\left(1-\frac{1}{h_{0}}\right) \frac{e^{-2 \alpha}}{\alpha}+\mathcal{O}\left(\frac{e^{-3 \alpha}}{\alpha}\right) .
$$

This immediately implies (2.16).

Remark 2.7. Since $E^{\mathrm{a}}$ and $E^{\mathrm{scb}}$ are Fréchet differentiable in suitable function spaces it should be possible, using nonlinear analysis techniques such as the inverse function theorem, to extend the results from the linearized model problem to the fully nonlinear problem, provided that the stiffness parameter $\alpha$ is sufficiently large. Techniques of this kind have been used, for example, in [37]. 


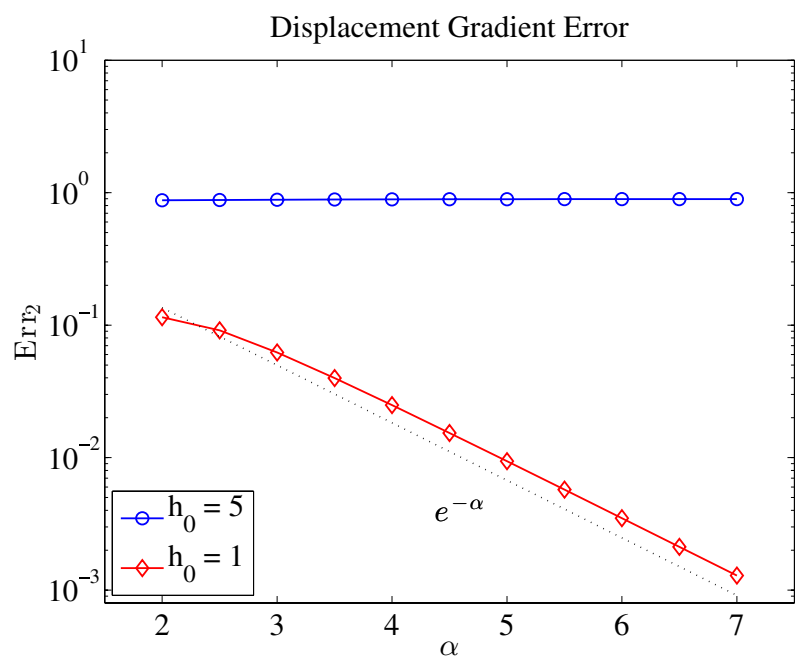

Figure 3. Relative error in the $W^{1,2}$-seminorm of the 1D nonlinear SCB model for varying stiffness parameter $\alpha$ and two types of finite element grids; $c f$. Section 2.5 .

\subsection{Numerical results}

We confirm through numerical experiments that the results of Propositions 2.5 and 2.6 are still valid in the nonlinear setting. In these experiments we choose $r_{0}=1$ instead of (2.4), choose a finite chain with 31 atoms, and let $\alpha$ vary between 2 and 7. For experiments with $h_{0}=5$ the gridpoints for the Cauchy-Born and SCB models are chosen as $X_{h}=(0,5,10, \ldots, 30)$. For experiments with $h_{0}=1$, the gridpoints are chosen as $X_{h}=(0,1,5, \ldots, 25,29,30)$.

For each model, the associated energy is minimized over the associated admissible space: the space $\mathrm{P}_{0}\left(X_{h}\right)$ for the CB and SCB models, and the space $\mathrm{P}_{0}(\{0,1, \ldots, 30\})$ for the atomistic model.

The results of the experiments are displayed in Figures 3 and 4. All results except for the relative error in the mean strain with $h_{0}=1$ confirm our analytical results in the linearized case. We have, at present, no explanation why the mean strain error $\overline{\operatorname{Err}}$ with $h_{0}=1$ is of the order $\mathcal{O}\left(e^{-3 \alpha}\right)$ instead of the predicted $\mathcal{O}\left(e^{-2 \alpha}\right)$. An asymptotic analysis in the linearized case to higher order gives the expansion $\overline{\operatorname{Err}}=2 e^{-2 \alpha}+\mathcal{O}\left(e^{-3 \alpha}\right)$, and hence does not shed any light on this issue.

\section{NumericAl Results IN 2D}

In this section we investigate numerically, to what extent the $1 \mathrm{D}$ results might extend to the $2 \mathrm{D}$ setting. We will formulate a problem in a semi-infinite strip, where we anticipate relaxation only in the normal direction to the surfaces, and we therefore expect the same behaviour as in the 1D case. This is fully confirmed by the results of our numerical experiment.

\subsection{Formulation of the SCB method}

In $2 \mathrm{D}$ one expects (this is rigorously proven only for large stiffness parameter $\alpha[36]$ ) that the groundstate under Morse potential interaction is the triangular lattice. Hence we choose as the atomistic reference configuration a subset $\Lambda \subset \mathrm{A} \mathbb{Z}^{2}$, where

$$
A=\left[\begin{array}{cc}
1 & 1 / 2 \\
0 & \sqrt{3} / 2
\end{array}\right] .
$$




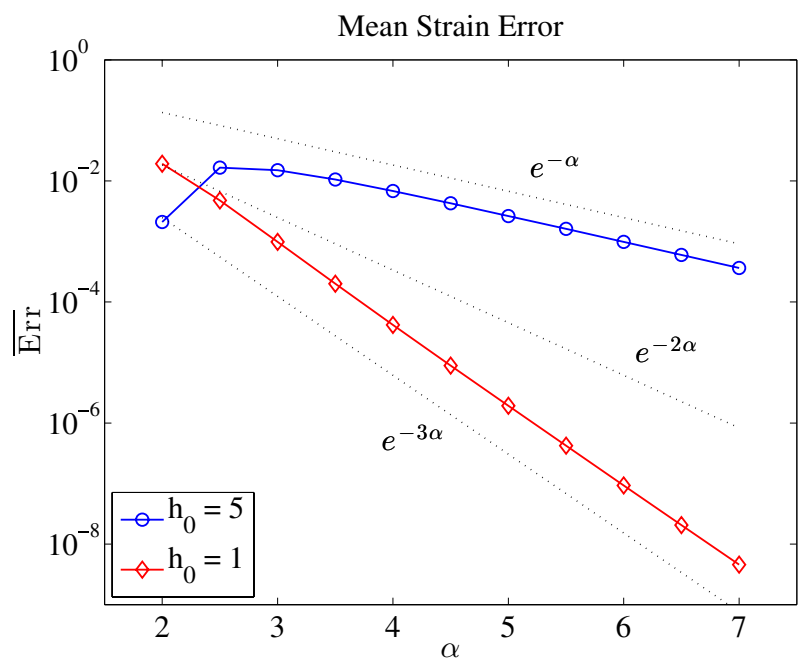

FiguRE 4. Relative error in the mean strain of the 1D nonlinear SCB model for varying stiffness parameter $\alpha$ and two types of finite element grids; $c f$. Section 2.5. We have no explanation at present why, in this experiment, the error is of a higher order $\mathcal{O}\left(e^{-3 \alpha}\right)$ than predicted by the linearized analysis $\mathcal{O}\left(e^{-2 \alpha}\right)$.

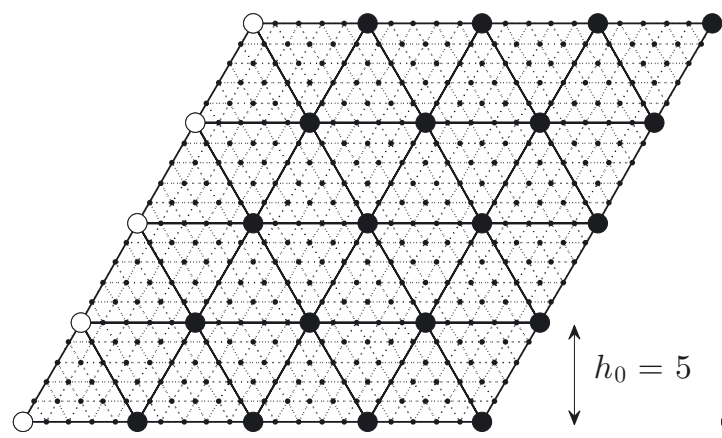

(a)

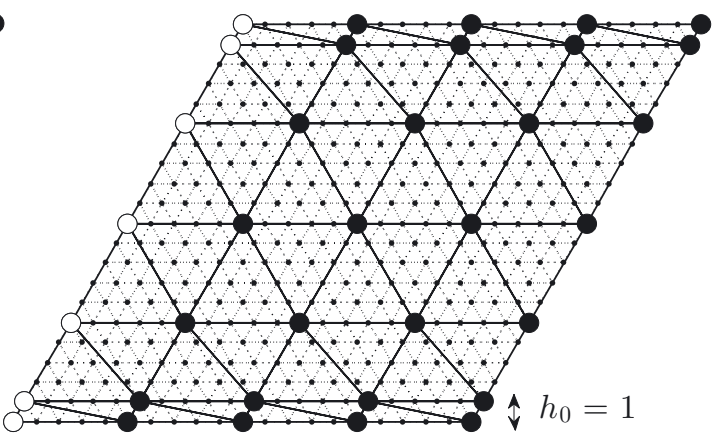

(b)

Figure 5. Computational domain used in the numerical experiment described in Section 3. The small disks denote the set $\Lambda$; the dotted grid is the micro-triangulation $\mathcal{T}_{\mathrm{a}}$; the the large black disks denote the finite element nodes; the large white discs denote finite element nodes that are periodically repeated; the black lines denote the macro-triangulation $\mathcal{T}_{h}$.

For future reference, we define $a_{1}:=(1,0), a_{2}:=(1 / 2, \sqrt{3} / 2)$ and $a_{3}:=(-1 / 2, \sqrt{3} / 2)$, which are the directions of nearest-neighbour bonds.

Specifically, we choose $N_{1}, N_{2} \in \mathbb{N}$ and define

$$
\Lambda:=\left\{\mathrm{A}\left(n_{1}, n_{2}\right)^{T} \in \mathrm{A} \mathbb{Z}^{2} \mid 1<n_{1} \leq N_{1}, 0 \leq n_{2} \leq N_{2}\right\},
$$

as the computatinal cell of the strip $\Lambda^{\#}:=\left\{\mathrm{A}\left(n_{1}, n_{2}\right)^{T} \in \mathrm{A} \mathbb{Z}^{2} \mid 0 \leq n_{2} \leq N_{2}\right\}$; cf. Figure 5 . The corresponding continuous domain is $\Omega:=\mathrm{A}\left(\left(0, N_{1}\right] \times\left(0, N_{2}\right]\right)$.

An admissible deformed configuration is a map $y: \Lambda^{\#} \rightarrow \mathbb{R}^{2}$, which is periodic in the $a_{1}$-direction, that is, $y\left(\xi+N_{1} a_{1}\right)=y(\xi)+N_{1} a_{1}$. 


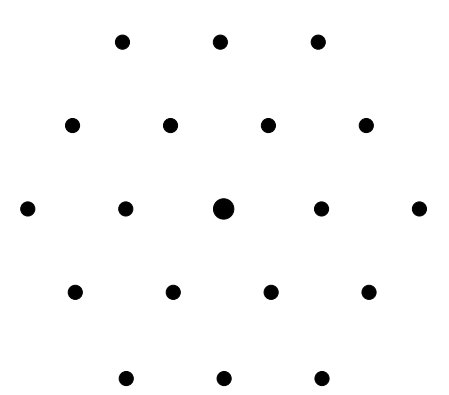

(a)

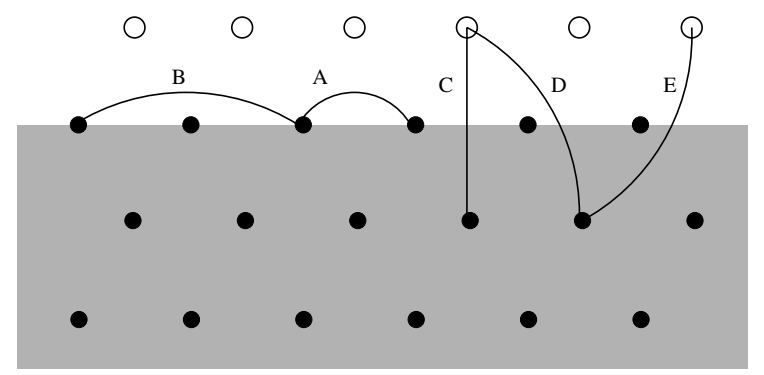

(b)

FiguRE 6. (a) Third interaction neighbourhood. (b) Construction of $\gamma$ : bonds A, B are underestimated by the Cauchy-Born approximation (counted only half), while the bonds C, $\mathrm{D}, \mathrm{E}$ are overestimated (they do not exist in the atomistic model but are counted half in the Cauchy-Born model).

For simplicity we consider only second-neighbour interactions (measured in hopping distance). For each $\xi \in \Lambda$ let $\mathcal{N}_{\xi}:=\left\{\eta \in \Lambda^{\#},|\eta-\xi| \leq 2\right\}$ denote the interaction neighbourhood of $\xi$, then the potential energy of a deformed configuration is given by

$$
E^{\mathrm{a}}(y):=\sum_{\xi \in \Lambda} \frac{1}{2} \sum_{\eta \in \mathcal{N}_{\xi}} \phi(|y(\eta)-y(\xi)|),
$$

where $\phi$ is again the Morse potential.

To evaluate the deformation gradient $\nabla y$ of a discrete deformation $y$, we note that $\Lambda^{\#}$ has a natural triangulation $\mathcal{T}_{\mathrm{a}}$ (see Fig. 5), and identify $y$ with its continuous piecewise affine interpolant in $\mathrm{P}_{1}\left(\mathcal{T}_{\mathrm{a}} ; \mathbb{R}^{2}\right)$.

Let $\mathcal{T}_{h}$ be a coarse triangulation of $\Omega$ (which can be repeated periodically) and let $\mathrm{P}_{1}\left(\mathcal{T}_{h} ; \mathbb{R}^{2}\right)$ denote the space of continuous and piecewise affine deformations of $\Omega$, such that $y_{h}\left(x+N_{1} a_{1}\right)=y_{h}(x)+N_{1} a_{1}$, then the SCB energy of a deformation $y_{h} \in \mathrm{P}_{1}\left(\mathcal{T}_{h} ; \mathbb{R}^{2}\right)$ is given by

$$
E^{\mathrm{scb}}\left(y_{h}\right)=\int_{\Omega} W\left(\nabla y_{h}\right) \mathrm{d} x+\int_{\Gamma} \gamma\left(\nabla y_{h}, \nu\right) \mathrm{d} x
$$

where $\Gamma \subset \partial \Omega$ denotes the free boundary, that is the portion of the boundary with normal $\nu= \pm(0,1), W$ is the Cauchy-Born stored energy function and $\gamma$ the SCB surface energy function, which are defined as follows:

- If we denote by $\mathcal{N}_{\mathrm{cb}}$ the interaction neighbourhood of the origin in the infinite lattice $\mathrm{A} \mathbb{Z}^{2}$ (see Fig. 6a), then the Cauchy-Born stored energy function is given by

$$
W(\mathrm{~F})=\frac{1}{\operatorname{det} \mathrm{A}} \sum_{\eta \in \mathcal{N}_{\mathrm{cb}}} \phi(|\mathrm{F} \eta|) .
$$

- To define $\gamma$, we assume throughout that all surfaces of $\Omega$ are aligned with one of the three directions $a_{1}, a_{2}$, or $a_{3}$, that is, $\nu \perp a_{j}=: \nu^{\perp}$. Then the requirement that the SCB energy is exact under homogeneous deformations, in domains without corners, yields the expression

$$
\gamma(\mathrm{F}, \nu)=\frac{1}{2} \phi\left(\left|\mathrm{F} \nu^{\perp}\right|\right)+\frac{1}{2} \phi\left(2\left|\mathrm{~F} \nu^{\perp}\right|\right)-\frac{1}{2} \phi(\sqrt{3}|\mathrm{~F} \nu|)-\frac{1}{2} \phi\left(2\left|\mathrm{FQ}_{12} \nu\right|\right)-\frac{1}{2} \phi\left(2\left|\mathrm{FQ}_{12}^{T} \nu\right|\right),
$$

where $Q_{12}$ denotes a rotation through arclength $2 \pi / 12$; see Figure $6 \mathrm{~b}$ for an illustration. A rigorous proof of this formula follows immediately from Shapeev's bond density lemma [34]; see also [30] for general results in this direction. 


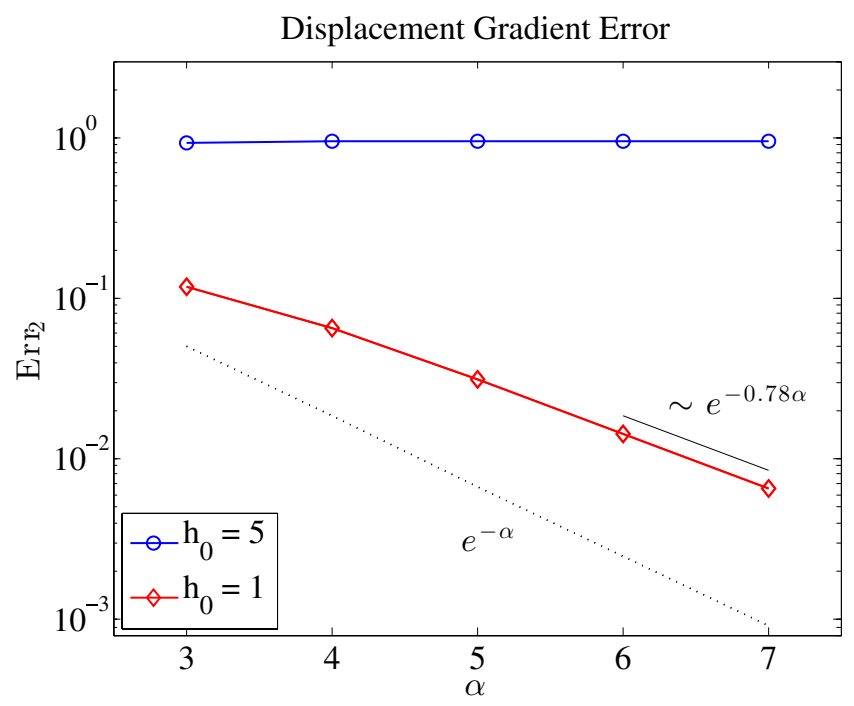

FiguRE 7. Relative error in the $W^{1,2}$-seminorm of the 2D SCB model in the flat interface example described in Section 3, for varying stiffness parameter $\alpha$ and two types of finite element grids.

\subsection{Numerical results}

In the numerical experiments we consider two types of finite element grids: a uniform grid with spacing $h=h_{0}=5$ (cf. Fig. 5a), and a grid with an additional layer of elements at the free boundary, atomic spacing $h_{0}=1$ in the normal direction and uniform spacing $h=5$ in the tangential direction ( $c f$. Fig. 5b). We will again measure the following relative errors:

$$
\operatorname{Err}_{2}:=\frac{\left\|\nabla y_{h}^{\mathrm{scb}}-\nabla y^{\mathrm{a}}\right\|_{L^{2}}}{\left\|\nabla y_{h}^{\mathrm{cb}}-\nabla y^{\mathrm{a}}\right\|_{L^{2}}}, \quad \text { and } \quad \overline{\operatorname{Err}}:=\frac{\left|\int_{\Omega}\left(\nabla y_{h}^{\mathrm{scb}}-\nabla y^{\mathrm{a}}\right) \mathrm{d} x\right|}{\left|\int_{\Omega}\left(\nabla y_{h}^{\mathrm{cb}}-\nabla y^{\mathrm{a}}\right) \mathrm{d} x\right|},
$$

where $y^{\mathrm{a}}, y_{h}^{\mathrm{scb}}$, and $y_{h}^{\mathrm{cb}}$ denote the minimizers of, respectively, $E^{\mathrm{a}}, E^{\mathrm{scb}}$, and $E^{\mathrm{scb}}$ with $\gamma=0$. That is, $\operatorname{Err}_{2}$ and

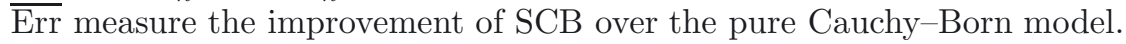

We remark, that the additional computational cost for the mesh with $h_{0}=1$ compared with $h_{0}=5$ is fairly moderate. Indeed, Figure 5 makes it clear that the extra cost scales with surface area. For surface dominated materials in $3 \mathrm{D}$ this would become more significant, but at worst only increase the cost by a moderate constant factor.

The numerical results are displayed in Figures 7 and 8. They do not as clearly display the predicted convergence rates, as in the $1 \mathrm{D}$ case. It cannot be concluded whether or not the errors approach these rates for increasing values of $\alpha$. However, it is again clear that the average strain has a much higher accuracy than the pointwise strain field, and that the additional mesh layer again substantially improves the accuracy of the method.

Moreover, we note the interesting fact that the numerical rates in the intervals $\alpha \in[6,7]$ in Figures 7 and 8 , while lower than predicted, are nevertheless consistent with each other in the following sense: the rates for $\operatorname{Err}_{2}$ with $h_{0}=1$ and for $\overline{\operatorname{Err}}$ with $h_{0}>1$ are approximately the same while the rate for $\overline{\text { Err }}$ with $h_{0}=1$ is doubled.

\section{Conclusion}

We presented an error analysis of the SCB method in the case where the dominant effect is surface relaxation in the normal direction. Our main results are: (1) we showed that a suitable "approximation parameter" is the 


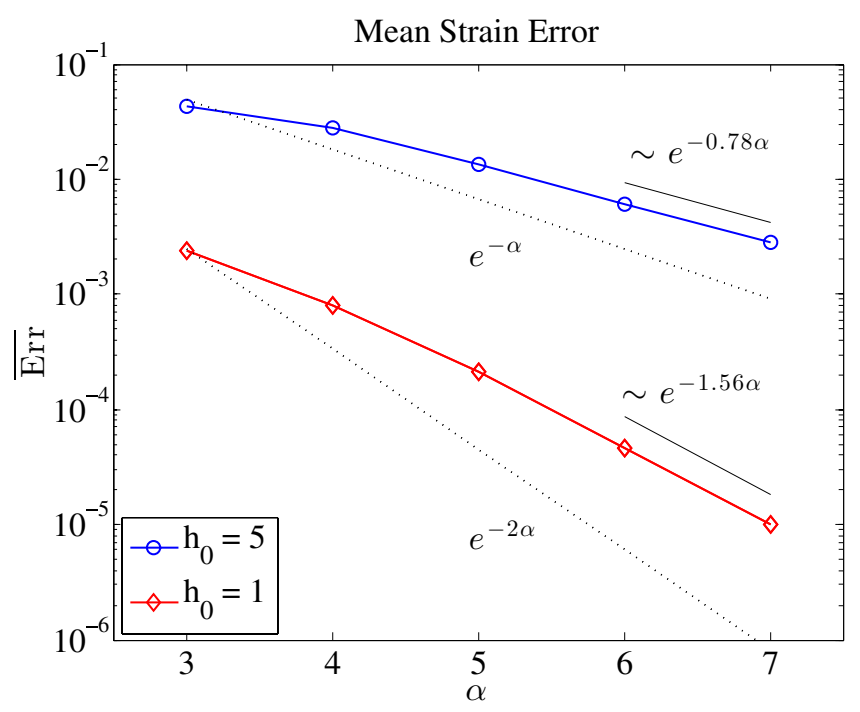

FiguRE 8. Relative error for the mean strain of the 2D SCB model applied to the flat interface example described in Section 3, for varying stiffness parameter $\alpha$ and two types of finite element grids.

stiffness of the interaction potential. (2) We showed that the mean strain (which is an important quantity of interest) has a much lower error than the strain field. (3) We showed that adding a single mesh layer at the free boundary with atomic spacing in the normal direction yields a substantial improvement to the accuracy of the SCB method with only moderate increase in the computational cost.

We also performed numerical experiments for domains with corners, which remain inconclusive so far. At corners there is an interplay between the normal stress and tangential stress of adjacent edges, which creates additional elastic fields. A finer analysis of this case is still required. In particular, it would be interesting to understand whether normal or tangential forces dominate the behaviour of the system in that case. Further corrections to the energy at corners may also be required, for example, as proposed by Rosakis [30].

Acknowledgements. We thank an anonymous referee for a detailed critique of our original manuscript and many useful suggestions that substantially improved the quality of this work.

\section{Appendix A. Proofs}

Proof of Propositions 2.1 and 2.2. For each $\ell \in \mathbb{N}$ we have

$$
\begin{aligned}
\phi\left(1+u_{\ell}\right)+\phi\left(2+u_{\ell}+u_{\ell+1}\right) & =\phi(1)+\phi^{\prime}\left(1+\theta_{\ell}^{(1)}\right) u_{\ell}+\phi(2)+\phi^{\prime}\left(2+\theta_{\ell}^{(2)}\right)\left(u_{\ell}+u_{\ell+1}\right) \\
& =\phi^{\prime}\left(1+\theta_{\ell}^{(1)}\right) u_{\ell}+\phi^{\prime}\left(2+\theta_{\ell}^{(2)}\right)\left(u_{\ell}+u_{\ell+1}\right),
\end{aligned}
$$

where $\left(\theta_{\ell}^{(j)}\right)_{\ell \in \mathbb{N}} \in \ell^{1}$ by Taylor's theorem, and we used the fact that $\phi(1)+\phi(2)=W(1)=0$. Since $\ell^{1} \subset \ell^{\infty}$, it follows that the coeffcients $\phi^{\prime \prime}\left(j+\theta_{\ell}^{(j)}\right)$ are bounded independently of $\ell$. Thus we deduce that $\ell \mapsto \phi\left(1+u_{\ell}\right)+$ $\phi\left(2+u_{\ell}+u_{\ell+1}\right)$ belongs to $\ell^{1}$ and hence $E^{\mathrm{a}}: \ell^{1} \rightarrow \mathbb{R}$ is well-defined.

Repeating the foregoing argument for a perturbation from a general state $u \neq 0$, but expanding to second or third order, establishes the Fréchet differentiability of $E^{\mathrm{a}}$. Here, we also need to use the embeddings $\ell^{1} \subset \ell^{2} \subset \ell^{3}$.

The same argument can be applied to prove Proposition 2.2. (Since the mesh size satisfies $h_{j} \geq 1$ the embedding $\ell_{h}^{1} \subset \ell_{h}^{p}$ for $p \geq 1$ holds again in this case). 
Proof of Proposition 2.3. Inserting the definition of $r_{0}$ from (2.4) into $\phi^{\prime \prime}(1)$ yields

$$
\phi^{\prime \prime}(1)=4 \alpha^{2} e^{-2 \alpha\left(1-r_{0}\right)}-2 \alpha^{2} e^{-\alpha\left(1-r_{0}\right)}=4 \alpha^{2}\left(\frac{1+2 e^{-\alpha}}{1+2 e^{-2 \alpha}}\right)^{2}-2 \alpha^{2}\left(\frac{1+2 e^{-\alpha}}{1+2 e^{-2 \alpha}}\right) .
$$

Expanding

$$
\frac{1+2 e^{-\alpha}}{1+2 e^{-2 \alpha}}=1+2 e^{-\alpha}+\mathcal{O}\left(e^{-2 \alpha}\right)
$$

we obtain

$$
\phi^{\prime \prime}(1)=4 \alpha^{2}\left(1+4 e^{-\alpha}\right)-2 \alpha^{2}\left(1+2 e^{-\alpha}\right)+\mathcal{O}\left(\alpha^{2} e^{-2 \alpha}\right)=2 \alpha^{2}+12 \alpha^{2} e^{-\alpha}+\mathcal{O}\left(\alpha^{2} e^{-2 \alpha}\right) .
$$

Similar calculations yield the expansions

$$
\begin{aligned}
\phi^{\prime}(2) & =2 \alpha e^{-\alpha}+2 \alpha e^{-2 \alpha}+\mathcal{O}\left(\alpha e^{-3 \alpha}\right), \quad \text { and } \\
\phi^{\prime \prime}(2) & =-2 \alpha^{2} e^{-\alpha}+\mathcal{O}\left(\alpha^{2} e^{-3 \alpha}\right) .
\end{aligned}
$$

Writing out $U_{0}^{\text {scb }}$ in terms of the Morse potential, and using the fact that $2 \leq 4-2 / h_{0} \leq 4$, which ensures that $\phi^{\prime \prime}(1)+\left(4-2 / h_{0}\right) \phi^{\prime \prime}(2) \geq W^{\prime \prime}(1)>0$, we obtain

$$
\begin{aligned}
h_{0} U_{0}^{\mathrm{scb}} & =\frac{\phi^{\prime}(2)}{\phi^{\prime \prime}(1)+\left(4-2 / h_{0}\right) \phi^{\prime \prime}(2)}=\frac{\phi^{\prime}(2)}{\phi^{\prime \prime}(1)} \frac{1}{1+\left(4-2 / h_{0}\right) \frac{\phi^{\prime \prime}(2)}{\phi^{\prime \prime}(1)}} \\
& =\frac{\phi^{\prime}(2)}{\phi^{\prime \prime}(1)}\left[1-\left(4-\frac{2}{h_{0}}\right) \frac{\phi^{\prime \prime}(2)}{\phi^{\prime \prime}(1)}+\mathcal{O}\left(\left(\frac{\phi^{\prime \prime}(2)}{\phi^{\prime \prime}(1)}\right)^{2}\right)\right] .
\end{aligned}
$$

Inserting the expansions (A.2) to (A.4) gives (2.11).

To prove (2.12) we first expand $\lambda$ in terms of $\beta:=\frac{\phi^{\prime \prime}(2)}{\phi^{\prime \prime}(1)}$, and then in terms of $e^{-\alpha}$,

$$
\begin{aligned}
\lambda & =\frac{1}{2 \beta}(\sqrt{1+4 \beta}-1-2 \beta) \\
& =\frac{1}{2 \beta}\left(1+\frac{1}{2}(4 \beta)-\frac{1}{8}(4 \beta)^{2}+\frac{1}{16}(4 \beta)^{3}+\mathcal{O}\left(\beta^{4}\right)-1-2 \beta\right) \\
& =-\beta+2 \beta^{2}+\mathcal{O}\left(\beta^{3}\right)=e^{-\alpha}-4 e^{-2 \alpha}+\mathcal{O}\left(e^{-3 \alpha}\right) .
\end{aligned}
$$

Inserting this result into (2.10) and a brief computation yield

$$
u_{0}^{\mathrm{a}}=\frac{\phi_{2}^{\prime}}{\phi_{1}^{\prime \prime}+\phi_{2}^{\prime \prime}(1+\lambda)}=\frac{e^{-\alpha}}{\alpha}-4 \frac{e^{-2 \alpha}}{\alpha}+\mathcal{O}\left(\frac{e^{-3 \alpha}}{\alpha}\right) .
$$

Since $u_{\ell}^{\mathrm{a}}=u_{0}^{\mathrm{a}} \lambda^{-\ell}$ the result (2.12) follows easily.

\section{REFERENCES}

[1] X. Blanc, C. Le Bris and P.-L. Lions, From molecular models to continuum mechanics. Arch. Ration. Mech. Anal. 164 (2002) 341-381.

[2] X. Blanc, C. Le Bris and F. Legoll, Analysis of a prototypical multiscale method coupling atomistic and continuum mechanics. ESAIM: M2AN 39 (2005) 797-826.

[3] A. Braides and M. Cicalese, Surface energies in nonconvex discrete systems. Math. Models Methods Appl. Sci. 17 (2007) 985-1037.

[4] A. Braides, A.J. Lew and M. Ortiz, Effective cohesive behavior of layers of interatomic planes. Arch. Ration. Mech. Anal. 180 (2006) 151-182. 
[5] R.C. Cammarata, Surface and interface stress effects in thin films. Prog. Surf. Sci. 46 (1994) 1-38.

[6] S. Cuenot, C. Frétigny, S. Demoustier-Champagne and B. Nysten, Surface tension effect on the mechanical properties of nanomaterials measured by atomic force microscopy. Phys. Rev. B 69 (2004) 165410.

[7] J. Diao, K. Gall and M.L. Dunn, Surface-stress-induced phase transformation in metal nanowires. Nat. Mater. 2 (2003) $656-660$.

[8] M. Dobson and M. Luskin, An analysis of the effect of ghost force oscillation on quasicontinuum error. ESAIM: M2AN 43 (2009) 591-604.

[9] M. Dobson, M. Luskin and C. Ortner, Accuracy of quasicontinuum approximations near instabilities. J. Mech. Phys. Solids 58 (2010) 1741-1757.

[10] W. E and P. Ming, Cauchy-Born rule and the stability of crystalline solids: static problems. Arch. Ration. Mech. Anal. 183 (2007) 241-297.

[11] M. Farsad, F.J. Vernerey and H.S. Park, An extended finite element/level set method to study surface effects on the mechanical behavior and properties of nanomaterials. Int. J. Numer. Methods Eng. 84 (2010) 1466-1489.

[12] G. Friesecke and F. Theil, Validity and failure of the Cauchy-Born hypothesis in a two-dimensional mass-spring lattice. $J$. Nonlinear Sci. 12 (2002) 445-478.

[13] W. Gao, S.W. Yu and G.Y. Huang, Finite element characterization of the size-dependent mechanical behaviour in nanosystems. Nanotechnol. 17 (2006) 1118-1122.

[14] M.E. Gurtin and A. Murdoch, A continuum theory of elastic material surfaces. Arch. Ration. Mech. Anal. 57 (1975) $291-323$.

[15] J. He and C.M. Lilley, The finite element absolute nodal coordinate formulation incorporated with surface stress effect to model elastic bending nanowires in large deformation. Comput. Mech. 44 (2009) 395-403.

[16] A. Javili and P. Steinmann, A finite element framework for continua with boundary energies. part I: the two-dimensional case. Comput. Methods Appl. Mech. Eng. 198 (2009) 2198-2208.

[17] H. Liang, M. Upmanyu and H. Huang, Size-dependent elasticity of nanowires: nonlinear effects. Phys. Rev. B 71 (2005) R241-403.

[18] W. Liang, M. Zhou and F. Ke, Shape memory effect in Cu nanowires. Nano Lett. 5 (2005) 2039-2043.

[19] C. Ortner and F. Theil, Nonlinear elasticity from atomistic mechanics. E-prints arXiv:1202.3858v3 (2012).

[20] H.S. Park, Surface stress effects on the resonant properties of silicon nanowires. J. Appl. Phys. 103 (2008) 123504.

[21] H.S. Park, Quantifying the size-dependent effect of the residual surface stress on the resonant frequencies of silicon nanowires if finite deformation kinematics are considered. Nanotechnol. 20 (2009) 115701.

[22] H.S. Park and P.A. Klein, Surface Cauchy-Born analysis of surface stress effects on metallic nanowires. Phys. Rev. B 75 (2007) 085408.

[23] H.S. Park and P.A. Klein, A surface Cauchy-Born model for silicon nanostructures. Comput. Methods Appl. Mech. Eng. 197 (2008) 3249-3260.

[24] H.S. Park and P.A. Klein, Surface stress effects on the resonant properties of metal nanowires: the importance of finite deformation kinematics and the impact of the residual surface stress. J. Mech. Phys. Solids 56 (2008) $3144-3166$.

[25] H.S. Park, K. Gall and J.A. Zimmerman, Shape memory and pseudoelasticity in metal nanowires. Phys. Rev. Lett. 95 (2005) 255504 .

[26] H.S. Park, P.A. Klein and G.J. Wagner, A surface Cauchy-Born model for nanoscale materials. Int. J. Numer. Methods Eng. 68 (2006) 1072-1095.

[27] H.S. Park, W. Cai, H.D. Espinosa and H. Huang, Mechanics of crystalline nanowires. MRS Bull. 34 (2009) $178-183$.

[28] H.S. Park, M. Devel and Z. Wang, A new multiscale formulation for the electromechanical behavior of nanomaterials. Comput. Methods Appl. Mech. Eng. 200 (2011) 2447-2457.

[29] H.-G. Roos, M. Stynes and L. Tobiska, Robust numerical methods for singularly perturbed differential equations, Convectiondiffusion-reaction and flow problems, 2nd edition. Springer Series in Comput. Math. 24 (2008).

[30] P. Rosakis, Continuum surface energy from a lattice model. E-prints arXiv:1201.0712 (2012).

[31] L. Scardia, A. Schlömerkemper and C. Zanini, Boundary layer energies for nonconvex discrete systems. Math. Mod. Methods Appl. Sci. 21 (2011) 777-817.

[32] B. Schmidt. On the passage from atomic to continuum theory for thin films. Arch. Ration. Mech. Anal. 190 (2008) 1-55.

[33] J.-H. Seo, Y. Yoo, N.-Y. Park, S.-W. Yoon, H. Lee, S. Han, S.-W. Lee, T.-Y. Seong, S.-C. Lee, K.-B. Lee, P.-R. Cha, H.S. Park, B. Kim and J.-P. Ahn, Superplastic deformation of defect-free au nanowires by coherent twin propagation. Nano Lett. 11 (2011) 3499-3502.

[34] A.V. Shapeev, Consistent energy-based atomistic/continuum coupling for two-body potentials in one and two dimensions. Multiscale Model. Simul. 9 (2011) 905-932.

[35] C.Q. Sun, B.K. Tay, X.T. Zeng, S. Li, T.P. Chen, J. Zhou, H.L. Bai and E.Y. Jiang, Bond-order-bond-length-bond-strength (bond-OLS) correlation mechanism for the shape-and-size dependence of a nanosolid. J. Phys.: Condens. Matter 14 (2002) $7781-7795$.

[36] F. Theil, A proof of crystallization in two dimensions. Commun. Math. Phys. 262 (2006) 209-236.

[37] F. Theil, Surface energies in a two-dimensional mass-spring model for crystals. ESAIM: M2AN 45 (2011) 873-899.

[38] G. Yun and H.S. Park, A multiscale, finite deformation formulation for surface stress effects on the coupled thermomechanical behavior of nanomaterials. Comput. Methods Appl. Mech. Eng. 197 (2008) 3337-3350.

[39] G. Yun and H.S. Park, Surface stress effects on the bending properties of fcc metal nanowires. Phys. Rev. B 79 (2009) 195421.

[40] J. Yvonnet, H. Le Quang and Q.-C. He, An XFEM/level set approach to modelling surface/interface effects and to computing the size-dependent effective properties of nanocomposites. Comput. Mech. 42 (2008) 119-131. 